\title{
Poly(Lactic Acid) with Segmented Perfluoropolyether Enchainment
}

\author{
Dahlia Haynes ${ }^{1}$, Amit K. Naskar ${ }^{1}$, Akhilesh Singh, ${ }^{2}$ Chih-Chao Yang ${ }^{3}$, Karen J. Burg, ${ }^{3}$ \\ Michael Drews, ${ }^{2}$ Graham Harrison, ${ }^{4}$ and Dennis W. Smith Jr. ${ }^{1, *}$ \\ ${ }^{1}$ Department of Chemistry, ${ }^{2}$ School of Material Science and Engineering, ${ }^{3}$ Department of
} Bioengineering, ${ }^{4}$ and Department of Chemical and Biomolecular Engineering.

Center for Advanced Engineering Fibers and Films

Clemson University, Clemson, SC 29634

*Correspondence should be addressed to D.W.S. Jr. dwsmith@clemson.edu

Supplementary Information 
Table S1. Calculated Surface energies of PLA and PFPE/PLA copolymers

\begin{tabular}{|c|c|c|c|c|c|c|}
\hline \multicolumn{5}{|c|}{ Surface Energy (mN/m) of PLA and PFPE/PLA copolymer } \\
\hline & \multicolumn{5}{|c|}{ Rabel (Regression) } & \multicolumn{3}{c|}{ Kaelble (Average) } \\
\hline Polymer & Polar & Dispersive & Total & Polar & Dispersive & Total \\
\hline PLA & 8.2 & 26.8 & 35.0 & 9.3 & 28.6 & 37.9 \\
\hline FluoroPLA1 & 2.4 & 15.2 & 17.5 & 3.6 & 16.3 & 19.9 \\
\hline FluoroPLA5 & 1.2 & 14.5 & 15.6 & 1.7 & 16.2 & 17.9 \\
\hline FluoroPLA10 & 1.6 & 15.0 & 16.6 & 3.8 & 17.3 & 21.1 \\
\hline FluoroPLA20 & 2.4 & 14.3 & 16.8 & 4.9 & 17.1 & 22.0 \\
\hline
\end{tabular}




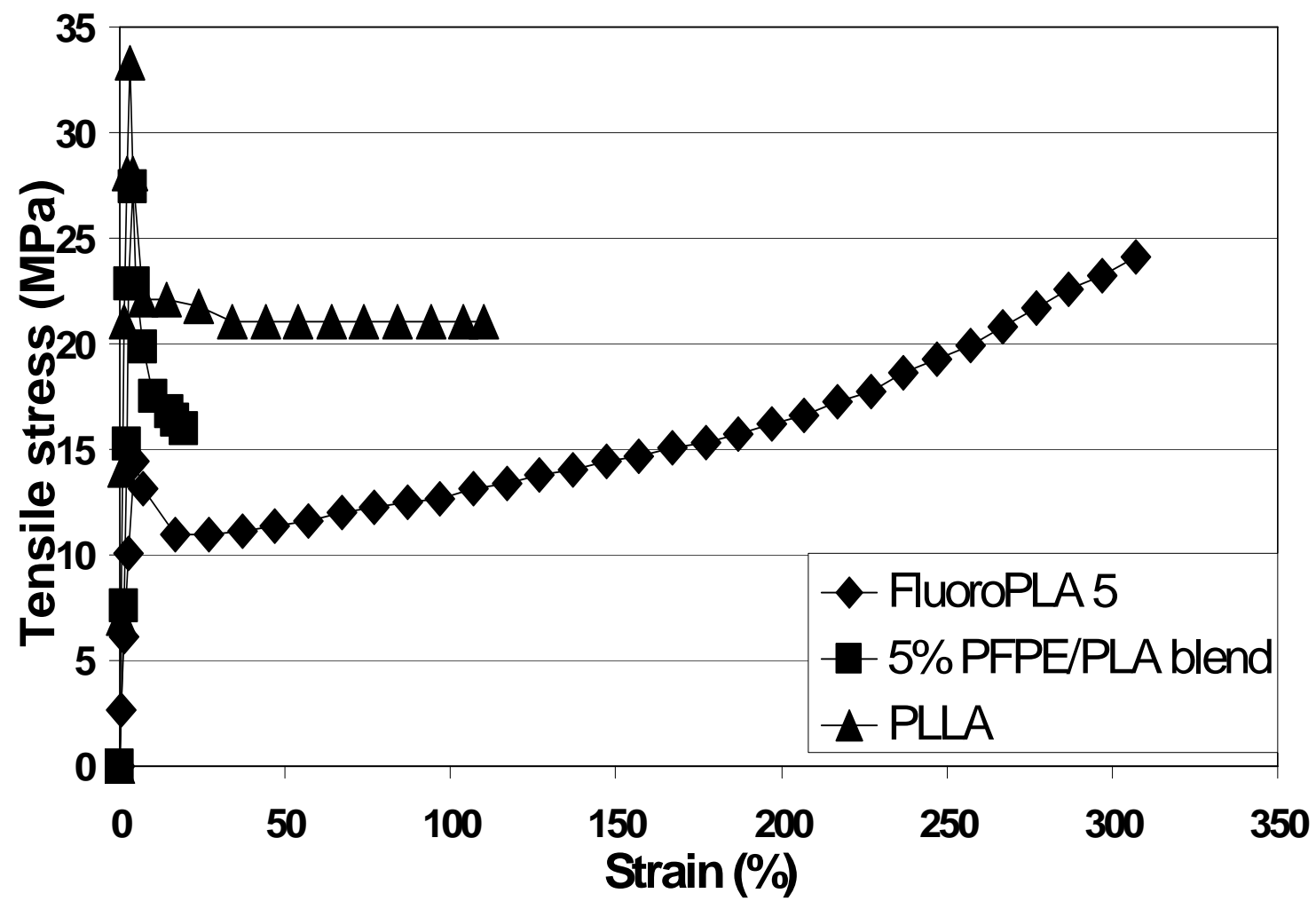

Figure S1. Stress-Strain curve of PLLA, FluoroPLA 5 and 5\% PFPE/PLA blend 